\title{
Potential analysis reveals changing number of climate states during the last $60 \mathrm{kyr}$
}

\author{
V. N. Livina ${ }^{1}$, F. Kwasniok ${ }^{2}$, and T. M. Lenton ${ }^{1}$ \\ ${ }^{1}$ School of Environmental Sciences, University of East Anglia, Norwich, UK \\ ${ }^{2}$ College of Engineering, Mathematics and Physical Sciences, University of Exeter, Exeter, UK
}

Received: 28 August 2009 - Published in Clim. Past Discuss.: 30 September 2009

Revised: 6 January 2010 - Accepted: 10 February 2010 - Published: 17 February 2010

\begin{abstract}
We develop and apply a new statistical method of potential analysis for detecting the number of states of a geophysical system, from its recorded time series. Estimation of the degree of a polynomial potential allows us to derive the number of potential wells in a system. The method correctly detects changes in the number of wells in artificial data. In ice-core proxy records of Greenland paleotemperature, a reduction in the number of climate states from two to one is detected sometime prior to the last glacial maximum (LGM), 23-19 kyr BP. This result is also found in analysis of Greenland Ca data. The bifurcation can be interpreted as loss of stability of the warm interstadial state of the DansgaardOeschger (DO) events. The proposed method can be applied to a wide range of geophysical time series exhibiting bifurcations.
\end{abstract}

\section{Introduction}

Knowing the number of states in a geophysical system is crucial for understanding its underlying dynamics and for modeling its behavior. Of particular interest are changes in the number of states which represent bifurcations of a system. Commonly the number of system states is inferred either by simply looking at time series data, or on the basis of theoretical models. Yet when there are two or more states present in a system the precise number is not always obvious from examining time series by eye (see e.g. Fig. 2b). In particular,

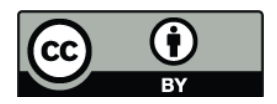

Correspondence to: V. N. Livina (v.livina@uea.ac.uk) what looks like a system with two states may actually contain more states. Consequently we have developed a more formal (though still necessarily approximate) method of estimating the number of system states from time series data.

In studies of paleoclimate changes, it is common to describe the glacial-interglacial cycles as shifts between different climate states. For example, one simple model (Paillard, 2001) assumes three (mild glacial, full glacial, interglacial) states for Northern Hemisphere ice volume. Within the last ice age, the Dansgaard-Oeschger events that are recorded in Greenland ice cores (Dansgaard et al., 1993), North Atlantic palaeoclimate records (Bond et al., 1997), and are now recognized further afield (Wang et al., 2001), are commonly treated as a two-state system (cold stadial, warm interstadial). The DO events are widely explained in terms of a model (Stommel, 1961; Rahmstorf, 1995; Ganopolski and Rahmstorf, 2001) for bistability of the Atlantic ocean thermohaline circulation, coupled to changes in sea-ice cover and atmospheric circulation. Potentially, different combinations of Northern Hemisphere ice volume and ocean-atmospheresea-ice state could give rise to multiple climate states as recorded in Greenland. Ice volume clearly affects the stability regime of the ocean-atmosphere-sea-ice as the Holocene interglacial has not exhibited the extreme DO variability seen in the last ice age.

Here we apply our new method to examine how many climate states are present in high-resolution Greenland Ice-Core Project (GRIP) (Dansgaard et al., 1993) and North Greenland Ice-Core Project (NGRIP) (NGRIP project, 2004) records, as a function of time through the last $60 \mathrm{kyr}$ (part of the last ice age and the Holocene). We also consider GRIP calcium data over the interval 60-11 kyr BP, which has annual resolution.

Published by Copernicus Publications on behalf of the European Geosciences Union. 
The precise mechanisms causing transitions between climate states do not concern us here. We make the simplest assumption that multiple climate states can be approximated by a non-oscillatory polynomial potential, albeit one that changes through time. Furthermore, we assume that transitions between climate states are triggered purely by stochastic noise. This is consistent with recent analysis of the DO events (especially in the GRIP and NGRIP records), which does not support any underlying periodicity (Ditlevsen et al., 2007).

\section{Dynamical framework}

We treat the climate system as a nonlinear dynamical system which can possess multiple states, with shifts between these different climate states induced by stochastic forcing. A one-dimensional conceptual model for this is given by the Langevin equation

$d z=-U^{\prime}(z) d t+\sigma d W$

where $U(z)$ is a potential function, $\sigma$ is the noise level and $W$ denotes a standard Wiener process. The state variable $z$ represents a large-scale climate variable like Greenland palaeotemperature and is here identified with ice-core proxy $\left(\delta^{18} \mathrm{O}\right.$ stable water isotope) records. In Ditlevsen (1999), the Fokker-Planck equation was used to derive the potential of the GRIP calcium record, with white non-Gaussian noise and two noise terms in the Langevin equation.

The shape of the potential is given by a polynomial

$U(z)=\sum_{i=1}^{L} a_{i} z^{i}$

where the order $L$ is even and the leading coefficient $a_{L}$ is positive for Eq. (1) to possess a stationary solution. The order of the polynomial controls the complexity of the potential. Increasing values of $L$ allow more states to be accommodated; for example, a fourth-order polynomial can capture a system with two states (double-well potential) (Kwasniok and Lohmann, 2009, 2010).

The number of system states is estimated by means of a polynomial fit of the probability density function of the data. Suppose the system is governed by Eq. (1). The corresponding Fokker-Planck equation for the probability density function $p(z, t)$

$\partial_{t} p(z, t)=\partial_{z}\left[U^{\prime}(z) p(z, t)\right]+\frac{1}{2} \sigma^{2} \partial_{z}^{2} p(z, t)$

has a stationary solution given by (Gardiner, 2004)

$p(z) \sim \exp \left[-2 U(z) / \sigma^{2}\right]$.

Given this one-to-one correspondence between the potential and the stationary probability density of the system, the potential can be reconstructed from time series data of the system as

$U=-\frac{\sigma^{2}}{2} \log p_{\mathrm{d}}$

where $p_{\mathrm{d}}$ is the empirical probability density of the data. This is estimated using a standard Gaussian kernel estimator (Silverman, 1986). We applied Matlab code with the built-in kdensity function and the NAG Fortran library (computing the Gaussian kernel density estimator using a fast Fourier transform). The estimator is

$\hat{f}(z)=\frac{1}{n h} \sum_{i=1}^{n} K\left(\frac{z-z_{i}}{h}\right)$,

where $K$ denotes the Gaussian kernel, $z_{i}$ are the data points, $n$ is the length of the data set and $h$ is the bandwidth controlling the smoothness of the estimator. Following Silverman (1986), we chose $h=1.06 s / n^{1 / 5}$, where $s$ is the standard deviation of the data set. Then least-square fits of $-\log p_{\mathrm{d}}$ weighted with the probability density of the data (Kwasniok and Lohmann, 2009) with polynomials of increasing even order $L$ are calculated, starting with $L=2$, until a negative leading coefficient $a_{L}$ is encountered. The polynomial of highest degree before first obtaining a negative leading coefficient is considered the most appropriate representation of the probability density of the time series, both locally and globally, avoiding overfitting of sampling fluctuations in the probability density.

The number of states $S$ in the system is then determined as

$S=1+\frac{I}{2}$,

where $I$ is the number of inflection points of the fitted polynomial potential of appropriate degree $L$ as described above. This definition takes into account not only the degree of the polynomial but its actual shape. We only look at even-order potentials with positive leading coefficient. These have positive curvature both at minus and plus infinity. Thus, inflection points can only occur in pairs (if any). Any potential has at least one state (with no inflection points). Then we count one further state for each pair of inflection points. This can be either a real minimum (well) or just a flattening in the potential corresponding to a degeneracy in the potential; definition (Eq. 7) accommodates both possibilities. The number of inflection points is numerically given as the number of sign changes in the second derivative on a fine enough mesh.

Examples of the polynomial fits for simulated data are given in Fig. 1. Given double-well potential data $\left(U(z)=z^{4}-2 z^{2}\right)$, the probability density of the time series has bimodal shape. The empirical potential given as $U=-\frac{\sigma^{2}}{2} \log p_{\mathrm{d}}$ can be fitted by polynomials of various orders, but only those of degree 2 and 4 have a positive leading coefficient. Higher-order polynomials have negative leading coefficients - although fitting the data better and better locally, they are clearly inadequate globally. The fourth-order 


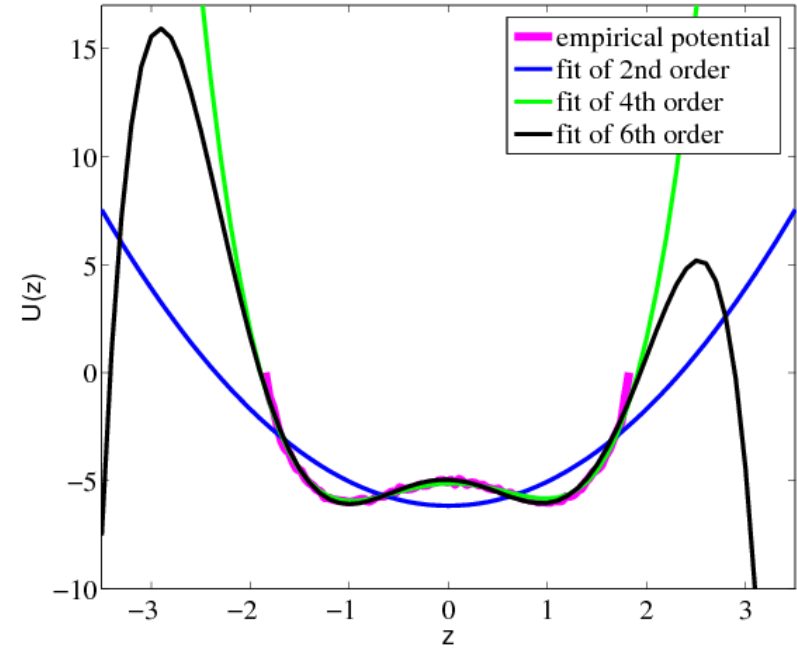

Fig. 1. Empirical potential (magenta curve) of double-well potential artifical data and its polynomial fits of $2 \mathrm{nd}$, 4th and 6th orders. The parabolic fit (2nd order) is not as accurate as the 4th-order polynomial, whereas the fit of 6 th order approximates the potential only locally, becoming negative for large $|z|$. The numerical algorithm chooses the highest order of polynomial that approximates the potential globally with positive leading coefficient; in this case it is the 4th-order polynomial. The method correctly classifies the data as double-well potential.

polynomial is the appropriate representation of the probability density and the method identifies the time series as double-well potential.

A simplified version of definition (Eq. 7) would be to only count the relative minima (real wells) in the potential. We opt here for the more comprehensive definition of system states as degenerate or nearly degenerate potentials have been shown to occur in the context of ice-core records (Kwasniok and Lohmann, 2009, 2010). Moreover, even if the system possesses real potential wells (for example, the artificial data shown in Fig. 2) changes in the number of states (bifurcations) tend to be picked up more quickly when using the inflection point definition as this is a more sensitive feature.

\section{Data}

To test the method for estimating the number of system states, we generated four sets of artificial data for four different potentials: one-well $\left(U(z)=z^{2}\right)$, double-well $(U(z)=$ $\left.z^{4}-2 z^{2}\right)$, triple-well $\left(U(z)=z^{6}-4.5 z^{4}+5 z^{2}\right)$ and four-well potential $\left(U(z)=z^{8}-6.5 z^{6}+13 z^{4}-8 z^{2}\right)$ (Fig. 2a). Simulated data of these systems were obtained by numerically integrating Eq. (1) using the Euler scheme. The noise level $\sigma=1.5$ was adopted, resulting in a mean state transition time (Kramers waiting time) similar to that occurring in the palaeoclimatic records when interpreting system time units as kyr. The sampling interval is 0.05 system time units. For each of the potentials, 150 time units worth of data were generated, resulting in 3000 data points. The subsets are
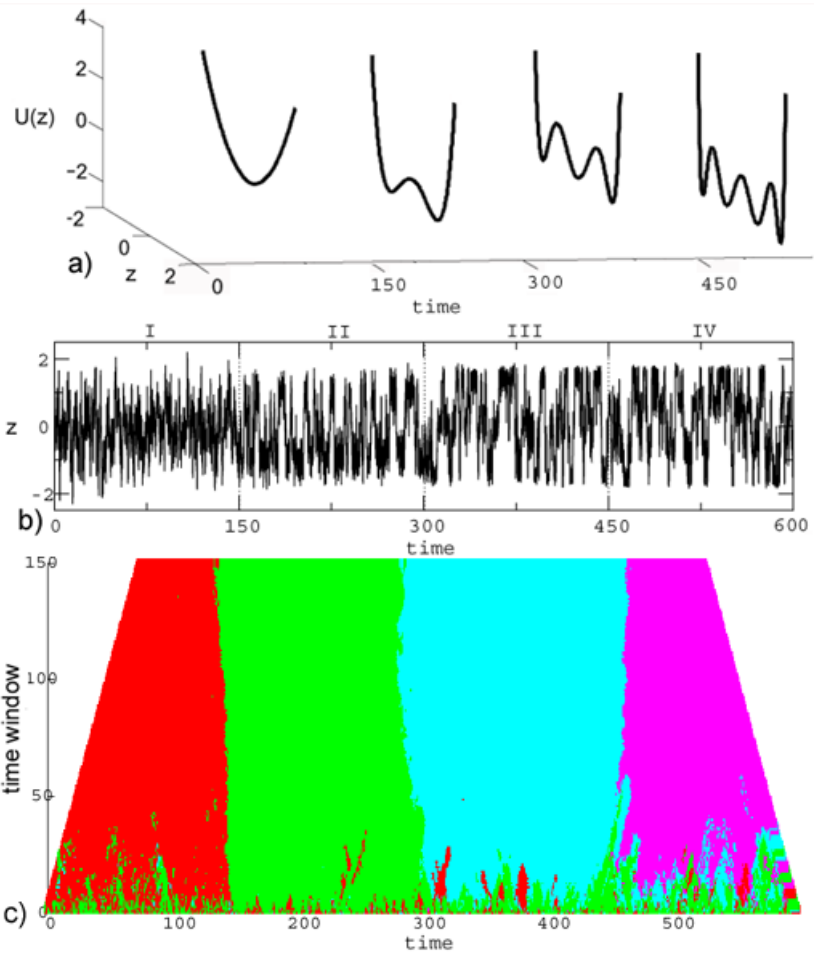

Fig. 2. Detection of the number of states in artificial data: (a) four different potentials with increasing number of wells from one to four; (b) the resulting time series; (c) contour plot of the number of detected states versus time and size of sliding window (red-one, green - two, cyan-three, purple-four). The number of detected states is mapped at the middle of the sliding time windows.

combined to produce a record with 12000 data points, corresponding to time running from 0 to 600 (Fig. 2b).

Having tested the methods on artificial data, we examined ice-core proxy records of palaeotemperature at two different sites $325 \mathrm{~km}$ apart in Greenland; GRIP (Dansgaard et al., 1993) (Fig. 3a) and NGRIP (NGRIP project, 2004) (Fig. 3c), on the most recent GICC05 time scale (Svensson et al., 2008). The data are $\delta^{18} \mathrm{O}$ stable water isotope records, which are a proxy for past air temperature at the ice-core sites. The records can also be influenced by changing water source temperatures and snowfall seasonality. There are significant differences between the two records during the last ice age, with NGRIP systematically colder (depleted in ${ }^{18} \mathrm{O}$ ), and more variable, when Northern Hemisphere ice sheets are more extensive (NGRIP project, 2004). This may be because NGRIP received a greater fraction of colder air coming over the northern side of the Laurentide ice sheet. Importantly, the difference between the two records does not show a component of millennial variability. Here we consider the last $60 \mathrm{kyr}$ NGRIP series with resolution $20 \mathrm{yr}$ and a part of the GRIP series (which is $112 \mathrm{kyr}$ long) in the same interval (60$0 \mathrm{kyr} \mathrm{BP}$ ) with the same temporal resolution of $20 \mathrm{yr}$. For the detection of changing numbers of states we use sliding windows of varying length through each dataset. 

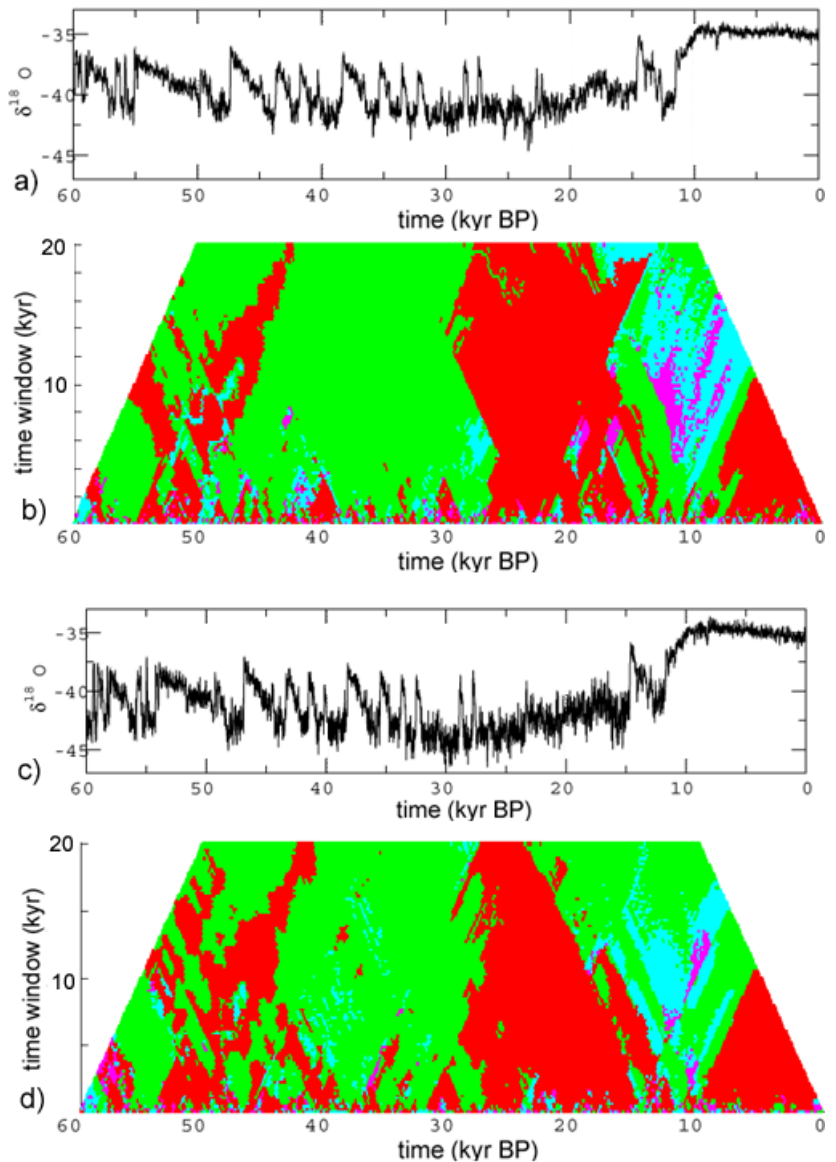

Fig. 3. GRIP ice-core data: (a) time series; (b) contour plot of the number of detected states versus time and size of sliding window, mapped at the middle of the sliding time windows (redone state, green - two, cyan - three, purple-four). NGRIP ice-core data: (c) time series; (d) contour plot of the number of detected states as in (b).

To further test initial results obtained on the $\delta^{18} \mathrm{O}$ data, we also examined $\mathrm{Ca}$ data from the GRIP project (Fig. 4) that has annual resolution and spans the interval 91-11 kyr BP. In our analysis, we consider a subset of this dataset starting at $60 \mathrm{kyr}$ BP to make it comparable with the other two datasets.

\section{Results}

We visualize the estimated number of system states as a colour contour plot, expressed as a function of the time at the middle of the data window (x-axis, aligned with the data time scale) and the time length of the data window (y-axis). As the results are plotted at the middle of their corresponding time windows, each point in the contour plot should be compared with the segment of the time series data centered at that particular point, and having the length of the corresponding time window.
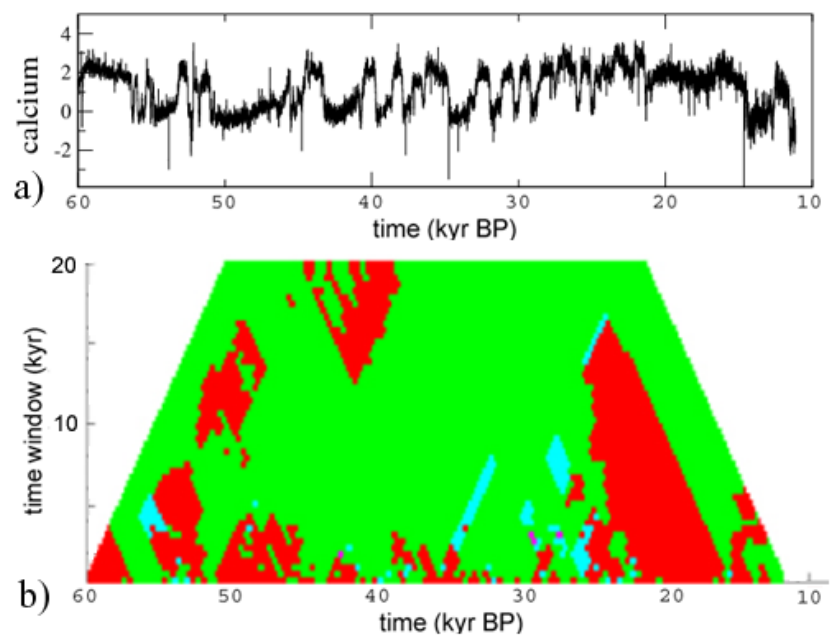

Fig. 4. GRIP calcium data: (a) time series; (b) contour plot of the number of detected states versus time and size of sliding window, mapped at the middle of the sliding time windows (red-one, green - two, cyan - three, purple-four).

In the artificial data, the number of states is generally identified correctly; changes are picked up quickly and reliably (Fig. 2c). For the smallest time windows, there are sporadic misidentifications of the number of states, due to poor statistics. Generally time windows of order 400 data points $(20$ time units) are sufficient to get reliable results.

The GRIP (Fig. 3a) and NGRIP (Fig. 3c) palaeotemperature data are highly variable and non-stationary. Nevertheless, the algorithm detects a number of interesting common features in both records (Fig. 3b, d). Over 60-25 kyr BP, two climate states are most commonly detected, consistent with the conventional view of the Dansgaard-Oeschger events. Exceptions of 1-state detection appear to be caused when long intervals of steady cooling, e.g. circa $55-50 \mathrm{kyr} \mathrm{BP}$, occupy a significant part of the analysis window.

The most pronounced feature, common to both records, is a change from 2-well to 1-well potential, generally detected by $25 \mathrm{kyr} \mathrm{BP}$ and inferred to have occurred somewhat prior to its detection. The transition is most sharply detected in the GRIP data. In the NGRIP data, the transition is less pronounced, consistent with the greater noise level in this dataset obscuring the detection of states. In both records, the shift to a 1-well potential is persistent, indicating a bifurcation in the climate system that occurred late in the last ice age but prior to the Last Glacial Maximum (LGM) 23-19 kyr BP (Yokoyama et al., 2000).

The return of a second state in the climate is only detected in both records, for most window sizes, around $12 \mathrm{kyr} \mathrm{BP}$ (i.e. at the end of the Younger Dryas), although it is sporadically detected earlier, from the time of the Bölling warming, especially in the NGRIP data. This reflects the growing influences of changes during the deglaciation on the algorithm. The detection of up to four states as the data window spans 
the interval of the last glacial termination is probably an artefact of the nonstationarity in the time series. Although these four climate states plausibly represent the LGM, BöllingAllerod warm interval, Younger Dryas cold interval, and the Holocene, it is perhaps more accurate to interpret the system as having two states about a moving trend.

As the end of the analysis window advances into the Holocene, the estimated number of system states declines. However, the number of climate states only reduces to one for both datasets when the analysis window comprises only the data since around $10 \mathrm{kyrBP}$, i.e. the start of the Holocene. For the GRIP data, there is some indication that the $8.2 \mathrm{kyr}$ BP event can influence the algorithm sufficiently that two climate states are detected when using small windows that span the event. The $8.2 \mathrm{kyr}$ BP event is also visible in the NGRIP data but has poorer signal-to-noise ratio, so does not exert the same influence on the algorithm.

Given that the ice-core time series are much shorter (in terms of the number of recorded transitions between states) than the simulated time series used above, the question arises how robust our results with the ice-core data are statistically. The mean recurrence time (Kramers waiting time) between DO events is $2.8 \mathrm{kyr}$ which is about the same as in the artificial data in nondimensional system time units. Hence the maximum window size of $20 \mathrm{kyr}$ in Fig. 3b, d corresponds to 20 units on the vertical axis in Fig. 2c. This is just enough to enable a correct detection of the number of states with very high probability. Together with the fact that our results are robust for a range of window sizes between 10 and $20 \mathrm{kyr}$ this gives us some confidence that our conclusions are reliable.

As a further test, we examined GRIP Ca data, and the result (Fig. 4) confirms the detected bifurcation in GRIP and NGRIP $\delta^{18} \mathrm{O}$, although the datasets have different origin and temporal resolution.

\section{Conclusions}

The results support the widely held view of the DansgaardOeschger events that they represent switches between 2 states of the climate system, which were present from before the start of our analysis $60 \mathrm{kyr}$ BP. Most interesting is the detection of a bifurcation in the climate system that reduced the number of states from 2 to 1 , sometime prior to the last glacial maximum (LGM) 23-19 kyr BP. This can be interpreted as the loss of a stable warm interstadial state. This result is not inconsistent with the conventional labelling of DO warming event 2 occurring at $23.4 \mathrm{kyr}$ BP (timing according to Rahmstorf, 2003) or the earlier DO events 3 at $27.8 \mathrm{kyr}$ BP and 4 at 29.0 kyr BP. Rather we suggest that although transitions to a warm state were still triggered up to the LGM, the resulting state became not even marginally stable. In the case of DO event 2 at least (and possibly 3 and even 4 ), the system was always destined to revert back to a cold state at a rate determined by its internal dynamics and/or the removal of some forcing factor. Indeed shortening of the duration of the warm interstadial state can be seen in the time series (Fig. 3a, c) as time progresses toward the LGM.

Clearly a warm state reappears during the deglaciation, in the form of the Bölling warming event (conventionally labelled DO event 1 at $14.6 \mathrm{kyr}$ BP; Rahmstorf, 2003), but whether it is appropriate to liken this to the earlier interstadials or the Holocene is unclear from our analysis. Likewise whether the Younger Dryas cool event should be likened to the earlier cold stadial state is ambiguous. Clearly Northern Hemisphere ice volume had declined significantly prior to either of these events, and was undergoing a transition from glacial to interglacial states. For the last $10 \mathrm{kyr}$ of the Holocene, under interglacial ice volume, only 1 stable Atlantic ocean-atmosphere-sea-ice state is detected. We interpret the $8.2 \mathrm{kyr}$ BP cooling event as a meltwater induced transient change in ocean-atmosphere-sea-ice from which the system was always destined to recover.

In this view the stability regime of the Atlantic oceanatmosphere-sea-ice system is reshaped and sometimes bifurcated by longer timescale changes in ice volume, as suggested by a number of models, e.g. (Ganopolski and Rahmstorf, 2001; Colin de Verdiere et al., 2006). Bistability of the Atlantic ocean-atmosphere-sea-ice system is present for intermediate Northern Hemisphere ice volumes, whereas under glacial maximum conditions there is only one stable cold state for Greenland temperature, and under interglacial conditions there is only a single (different) stable warm state.

The Langevin equation (Eq. 1) used in the present paper as a conceptual model is a fairly natural starting point which is already quite general but admittedly has limitations. The model is of first order; a higher-order model could accommodate richer dynamics. Sometimes, palaeoclimatic cycles are treated as oscillations, e.g. (Ghil et al., 1987). Our method can be readily extended to a second-order stochastically driven potential model supporting oscillatory behaviour (Kwasniok and Lohmann, 2010). Moreover, Eq. (1) does not allow for non-random external forcing, e.g. orbital forcing. Also the possibility of state-dependent noise rather than purely additive noise as well as coloured noise would be interesting to include. The proposed approach is initial, and further generalization of the method is to be developed.

Acknowledgements. We would like to thank J. Kantelhardt and Yu. Sapronov for discussions and P. Ditlevsen for useful comments on the manuscript. We are grateful to P. Ditlevsen for providing the GRIP Ca dataset for our analysis. The research was supported by NERC through project NE/F005474/1. We woud like to thank Michel Crucifix and an anonymous reviewer who helped us improve the manuscript.

Edited by: V. Masson-Delmotte 


\section{References}

Bond, G. Showers, W., Cheseby, M., Lotti, R., Almasi, P., de Menocal, P., Priore, P., Cullen, H., Hajdas, I., and Bonani, G.: A Pervasive Millennial-Scale Cycle in North Atlantic Holocene and Glacial Climates, Science, 278, 1257-1266, 1997.

Colin de Verdiere, A., Ben Jelloul, M., and Sevellec, F.: Bifurcation structure of thermohaline millennial oscillations, J. Climate, 19, 5777-5795, 2006.

Dansgaard, W., Johnson, S. J., Clausen, H. B., Dahljensen, D., Gundestrup, N. S., Hammer, C. U., Hvidberg, C. S., Steffensen, J. P., Sveinbjornsdottir, A. E., Jouzel, J., and Bond, G.: Evidence for general instability of past climate from a $250 \mathrm{kyr}$ ice-core record, Nature, 364, 218-220, 1993.

Ditlevsen, P. D.: Observation of $\alpha$-stable noise induced millennial climate changes from an ice-core record, Geophys. Res. Lett., 26(10), 1441-1444, 1999.

Ditlevsen, P. D., Andersen, K. K., and Svensson, A.: The DOclimate events are probably noise induced: statistical investigation of the claimed 1470 years cycle, Clim. Past, 3, 129-134, 2007 , http://www.clim-past.net/3/129/2007/.

Ganopolski, A. and Rahmstorf, S.: Rapid changes of glacial climate simulated in a coupled climate model, Nature, 409, 153158,2001

Gardiner, C. W.: Handbook of Stochastic Methods for Physics, Chemistry and the Natural Sciences (Springer Series in Synergetics), Springer, 3rd Edn., 415 pp., 2004.

Ghil, M., Mullhaupt, A., and Pestiaux, P.: Deep water formation and Quaternary glaciations, Clim. Dynam., 2, 1-10, 1987.

Kwasniok, F. and Lohmann, G.: Deriving dynamical models from paleoclimatic records: Application to glacial millennial-scale climate variability, Phys. Rev. E, 80, 066104, doi:10.1103/PhysRevE.80.066104, 2009.
Kwasniok, F. and Lohmann, G.: A stochastic nonlinear oscillator model for glacial millennial-scale climate transitions derived from ice-core data, Nonl. Proc. Geophys., submitted, 2010.

North Greenland Ice Core Project members: High-resolution record of Northern Hemisphere climate extending into the last interglacial period, Data are available at: http://www.iceandclimate. nbi.ku.dk/data/, Nature, 431, 147-151, 2004.

Paillard, D.: Glacial cycles: toward a new paradigm, Rev. Geophys., 39(3), 325-346, 2001.

Rahmstorf, S.: Bifurcation of the Atlantic thermohaline circulation in response to changes in the hydrological cycle, Nature, 378, 145-149, 1995.

Rahmstorf, S.: Timing of abrupt climate change: A precise clock, Geophys. Res. Lett., 30(10), 1510, doi:10.1029/2003GL017115, 2003.

Silverman, B. W.: Density estimation for statistics and data analysis, Chapman and Hall, 1986.

Stommel, H.: Thermohaline convection with two stable regimes of flow, Tellus, XIII, 224-230, 1961.

Svensson, A., Andersen, K. K., Bigler, M., Clausen, H. B., DahlJensen, D., Davies, S. M., Johnsen, S. J., Muscheler, R., Parrenin, F., Rasmussen, S. O., Röthlisberger, R., Seierstad, I., Steffensen, J. P., and Vinther, B. M.: A 60000 year Greenland stratigraphic ice core chronology, Clim. Past, 4, 47-57, 2008, http://www.clim-past.net/4/47/2008/.

Wang, Y. J., Cheng, H., Edwards, R. L., An, Z. S., Wu, J.Y., Shen, C. C., and Dorale, J. A.: A High-Resolution Absolute-Dated Late Pleistocene Monsoon Record from Hulu Cave, China, Science, 294, 2345-2348, 2001

Yokoyama Y., Lambeck, K., DeDekker, P., Johnston, P., and Fifield, L. K.: Timing of the Last Glacial Maximum from observed sealevel minima, Nature, 406, 713-716, 2000. 